ISSN: 2386-3919 - e-ISSN: 2386-3927

DOI: https://doi.org/10.14201/et201735297108

\title{
CARACTERIZACIÓN DE ESTILOS DE APRENDIZAJE EN ESTUDIANTES DE INGENIERÍA DE JORNADAS DIURNAS Y VESPERTINAS
}

\section{Learning styles differences among engineering students of daytime and evening classes programs}

\author{
Marco Antonio DíAz DíAZ* e Isabel OrTEGa SÁNCHEZ*** \\ UNED \\ Correo-e: *marcodiaz@gmail.com \\ *iortega@edu.uned.es
}

Recibido: 30-11-2016; Aceptado: 20-6-2017; Publicado: 30-11-2017

Ref. Bibl. MARCO ANTONIO DÍAZ DÍAZ e ISABEL ORTEGA SÁNCHEZ. Caracterización de estilos de aprendizaje en estudiantes de ingeniería de jornadas diurnas y vespertinas. Enseñanza \& Teaching, 35, 2-2017, 97-108.

RESUMEN: En esta investigación exploratoria se abordó el problema de determinar si existían diferencias en los estilos de aprendizaje entre los estudiantes de jornadas diurna y vespertina de una muestra de tres carreras de ingeniería de la Universidad Mayor. Esta inquietud surgió como una forma de comenzar a comprender el sustento de la observación que estudiantes con diferentes exigencias curriculares para el ingreso, frente a exigencias académicas similares, logran tener igual desempeño académico. En este estudio se usó una metodología cuantitativa con elementos descriptivos e inferenciales, encontrándose que en las tres carreras de la muestra estudiada la variable estilos de aprendizaje no presenta diferencias significativas entre estudiantes de las jornadas diurna y vespertina. A partir de este resultado se plantean nuevas interrogantes, principalmente, en torno a los mecanismos de ingreso a la educación universitaria en general y a las motivaciones y formas de enfrentar el estudio entre los estudiantes de jornada vespertina en la educación superior.

Palabras clave: estilos de aprendizaje; motivaciones; estudiantes de ingeniería; programas diurnos y vespertinos; desempeño académico. 
MARCO ANTONIO DÍAZ DÍAZ E ISABEL ORTEGA SÁNCHEZ

CARACTERIZACIÓN DE ESTILOS DE APRENDIZAJE EN ESTUDIANTES DE INGENIERÍA DE JORNADAS DIURNAS Y VESPERTINAS

SUMMARY: The purpose of this exploratory research was to identify if there were any differences in the student's learning styles of three engineering academic programs from daytime and evening classes. The motivation aroused from the observation that the academic performance of these two populations, in response to equally demanding academic programs, was equivalent, despite having far less demanding requirements for the acceptance in the evening class program. The results of this research showed no relevant differences in the learning styles of the students of the two programs, giving rise to more questions than answers, mainly regarding the motivations of the evening class students and the academic requirements for the acceptance in a tertiary institution.

Key words: learning styles; motivations; engineering students; daytime and evening academic programs; academic performance.

\section{INTRODUCCIÓN}

En nuestra sociedad se han ido imponiendo los conceptos de equidad en educación y de educación de calidad. Ambos conceptos suenan atractivos, sobre todo en conjunto, sin embargo, por esto mismo, conviene detenerse brevemente para meditar acerca de su significado.

La referencia a equidad hace mención a una disposición del ánimo que mueve a dar a cada uno lo que merece y a una moderación en el actuar con el prójimo. Es así como desde la perspectiva de las ciencias sociales, y en particular desde la educación, la equidad tiene varias dimensiones, entre las que se encuentran: inclusión, igualdad de oportunidades, movilidad social y vulnerabilidad. Planteamientos como estos se reflejan en el ámbito de la educación de nuestro país, como por ejemplo en la definición de la equidad como un concepto fundado «en el derecho de cada persona a contar con una educación de calidad que le permita desarrollar plenamente su potencial, independientemente de su origen socioeconómico, género, etnia u otras características que puedan ser fuente de discriminación" (Jiménez, Lagos y Durán, 2011: 22). Esto aplicado al ámbito de la educación superior implica, como lo ha planteado la OECD (2009):

[...] además de aumentar las vacantes del sistema, evaluar si los estudiantes de diferentes grupos de la sociedad chilena tienen un acceso igual y justo a la educación terciaria, significa evaluar no solo si obtienen y hacen uso de las vacantes en la educación superior -y luego se gradúan con éxito- sino también a qué tipo de institución terciaria asisten y si es el tipo, e institución de su elección (p. 78).

Así, se puede apreciar que el concepto de equidad en educación superior está, entre otros, fuertemente asociado al de calidad, la que tiene varias aristas. A partir de esta perspectiva se comenzó a investigar sobre cómo aprendían los estudiantes en diferentes carreras en distintas Facultades de la Universidad Mayor (UM), como una forma de contribuir a la comprensión de los procesos involucrados en el aprendizaje en el ámbito de una universidad privada. Esta información puede 
servir de base para adecuar las metodologías de enseñanza a las necesidades de los estudiantes dentro del contexto del desarrollo de competencias y del cambio de foco del proceso de enseñanza-aprendizaje, centrándolo en el estudiante (De Miguel, 2005).

Esta inquietud es la motivación tras la investigación necesaria para sustentar la tesis doctoral Estilos de aprendizaje y métodos pedagógicos en educación superior, investigación de cuyo tronco se desprende la rama que motiva este artículo.

A raíz de las conversaciones necesarias para implementar esta investigación en distintas facultades de la UM, surgió la observación que en una de ellas los estudiantes de una carrera de jornada vespertina tenían un rendimiento académico similar al de los estudiantes de la misma carrera pero de jornada diurna. Considerando que las exigencias académicas y curriculares eran comunes para ambas jornadas pero que existían diferencias en el sistema de ingreso producto de las diferencias en las vías y los requisitos, surgió la interrogante sobre ¿qué hacían distinto los estudiantes vespertinos que compensaba las diferencias y equiparaba el rendimiento?

Dicha consideración adquiere relevancia especialmente cuando en el ámbito de la educación superior subsisten prejuicios y paradigmas arraigados como, por ejemplo, los ligados a los requisitos de ingreso y trayectoria académica. Una pregunta que permite contextualizar la pregunta del párrafo anterior fue: ¿Existen diferencias en la forma de enfrentar el aprendizaje entre los estudiantes universitarios de jornadas diurna y vespertina de una carrera?

Ésta es una inquietud no menor, pues en Chile la población de estudiantes cursando educación superior el año 2013, en sus tres variantes, está estimada en aproximadamente 1.115 .000 personas (SIES/MINEUC, 2013). De éstos, según la misma fuente, el número total de estudiantes cursando jornada vespertina bordea el 26.7\%, es decir, aproximadamente 300.000 estudiantes. Según otro estudio (CNED, 2011), los estudiantes universitarios cursando jornada vespertina serían alrededor del 5\% del total, es decir, alrededor de 56.000 alumnos.

Otro factor a tener en cuenta es la distribución etárea puesto que aproximadamente un $60 \%$ de los estudiantes universitarios de jornada diurna se encuentra en el rango de 18 a 21 años, en cambio, la población universitaria asistente a jornada vespertina presenta una distribución etárea bimodal con predominio amplio de edades por sobre los 26 años (Failla, 2010).

Lo anterior predispone a suponer que estos estudiantes trabajan y estudian al mismo tiempo y que se ven en la necesidad de especializarse o de adquirir nuevos conocimientos y competencias con el objeto de tener mejores expectativas laborales en una sociedad cada vez más exigente y competitiva, pero en la que el acceso a la educación superior está cada vez más segmentado (Muñoz y Redondo, 2013).

\section{FUNDAMENTACIÓN}

La implementación en la um del modelo basado en el enfoque de competencias ha tenido por consecuencia el que la atención del proceso de enseñanza-aprendizaje 
se traslada al estudiante, con el fin de que éste adquiera el hábito de aprender mediante una búsqueda personal del conocimiento (De Miguel, 2005).

Entre los desafíos que este cambio plantea está el llegar a conocer cómo aprenden quienes son objeto de la enseñanza que se imparte, de manera que quienes transmiten conocimiento y experiencia, además de ayudar a orientar la búsqueda personal del estudiante, puedan, a su vez, adecuar las metodologías a las necesidades curriculares y a las formas de aprendizaje predominantes entre los estudiantes que asisten al curso. Otro beneficio más global que aporta este conocimiento es la posibilidad de establecer una sintonía entre el currículo propugnado para una carrera y los estilos de aprendizaje dominantes entre los estudiantes de esa carrera. Ésta es una forma de abordar el problema sobre equidad y calidad en educación superior planteado en un comienzo, pues así se engloban los procesos tanto de transmisión de conocimientos y experiencia como los de adquisición de competencias y personalización del aprendizaje, contextualizado a la realidad nacional.

Una de las manifestaciones de la crisis de calidad que se vive en la educación superior en Chile son las altas tasas de repitencia y abandono por parte de los estudiantes una vez que han ingresado a ésta, fenómeno que tendría un fuerte componente sociocultural pues la brecha en el logro académico entre dos individuos de diferente nivel socioeconómico (pecuniario y cultural) se acrecienta en la educación media, lo que dificulta el ingreso y la permanencia en la educación superior de los estudiantes de menor nivel socioeconómico (Muñoz y Redondo, 2013).

Las deficiencias en la calidad de la educación secundaria chilena, así como su inequidad, han sido ampliamente estudiadas (OECD, 2012; OECD, 2009), y se traducen, entre otras expresiones, en que la educación secundaria proporciona una mala base teórica y de hábitos de estudio, por lo que una alta proporción de los estudiantes que ingresan a la educación universitaria lo hacen sin haber tenido la oportunidad de desarrollar las competencias y actitudes necesarias para desenvolverse de manera efectiva y fluida en el ámbito de la educación superior.

Esta situación es más manifiesta entre los estudiantes provenientes de colegios municipalizados ya que éstos presentan una mayor tasa de repitencia y abandono que los provenientes de colegios particulares (OECD, 2012).

Como una forma de abordar esta inequidad, algunas universidades han implementado programas de cursos remediales para estudiantes con puntajes de PSU más bajos, para compensar las deficiencias en conocimientos al ingreso. Un problema que enfrenta este tipo de programas es que produce una sobrecarga académica sobre los estudiantes que lo requieran y que se encuentran en una situación desventajosa (OECD, 2009).

Otros elementos que tienen gran incidencia en el proceso de enseñanza-aprendizaje son el currículo, la docencia y las metodologías en uso, es decir, cómo los docentes realizan y evalúan la enseñanza, ya que la forma en que se llevan a cabo estas actividades se puede considerar como un reflejo de la actitud de las personas $y$, a su vez, la actitud se puede considerar como un constructo hipotético, susceptible de ser modificado y adecuado a la realidad contextual, aspecto central para cualquier adaptación. 
Así, en la docencia universitaria, la literatura da cuenta de la existencia de dos modos principales de enseñanza, que dan origen a dos modelos extremos y un tercero intermedio, éstos son: el modelo centrado en la enseñanza, transmisión de información y expositivo; el modelo centrado en el aprendizaje, facilitador del aprendizaje, interactivo; y modelos mixtos que se sitúan en una amplia zona intermedia, donde se puede dar una variada gama de combinaciones de elementos de ambos modelos principales, dependiendo éstas de lo que se ha dado en llamar habilidades docentes, las que a su vez son reflejo de las competencias de los profesores (Aguilera, 2012).

En este contexto, la aplicación del nuevo paradigma, que centra «el eje de la enseñanza sobre el aprendizaje autónomo del alumno» (De Miguel, 2005: 16), en particular a la educación universitaria en ciencias, ha resultado ser más compleja de lo previsto (Taber, 2009). Aún no se ha encontrado el equilibrio ni la forma que éste debe adoptar, entre la tarea del profesor y el aprendizaje autónomo del alumno para plasmar un aprendizaje significativo y de calidad, tal como lo reconoce Gallant (2011).

Es así como la enseñanza en ciencias, y en particular en salud, requiere estructurar el aprendizaje de manera que los estudiantes se beneficien de la experiencia y el conocimiento acumulados y no tengan que reeditar los descubrimientos de científicos y clínicos sobresalientes a lo largo de varios siglos, pues esta pretensión sería irreal (Taber, 2009).

No obstante, desde la perspectiva constructivista es importante que los alumnos procesen la nueva información recibida en términos del acervo de conocimiento relevante que poseen, de manera que, desde una perspectiva neurobiológica, se formen nuevas conexiones que subyazcan al desarrollo de nuevos marcos conceptuales de conocimiento y que éstos, a su vez, por una parte, se aproximen razonablemente al conocimiento establecido como deseable en el currículo (Taber, 2009) y, por otra, que esta capacidad de análisis e integración se transforme en un hábito aplicable a lo largo del ciclo de vida.

Los planteamientos anteriores son de carácter general, enmarcados en el cómo debe de ser el aprendizaje dentro de un paradigma, cuya implementación no ha estado exenta de problemas. Asi, tal como se hace aparente desde el conocimiento aportado por la neurobiología, hay formas de aprendizaje que requieren del reforzamiento por repetición (Spitzer, 2006), lo que puede ser calificado como conductista, por lo que hay etapas del proceso de enseñanza-aprendizaje que deben ser evaluadas cuidadosamente y que conducen a una aplicación ponderada de ambas tendencias, de acuerdo a la situación en ciernes y al aprendizaje esperado.

Un elemento que puede contribuir a encontrar un modo de concretar la forma de construir conocimiento dentro del marco que impone el aprendizaje de una profesión de carácter científico con elementos positivistas, como podrían ser las de Ingeniería y Medicina, es el que los participantes del proceso de enseñanza-aprendizaje tomen conciencia de los estilos de aprendizaje de los discentes, como una forma de contribuir a un proceso de aprendizaje más efectivo (Kolb, en Coffield, 
Moseley, Hall y Ecclestone, 2004) tanto desde el punto de vista de los conocimientos formales como de las competencias del sujeto.

En este contexto, según Kolb en Coffield et al. (2004), el aprendizaje es el proceso por el cual se crea el conocimiento a partir de la transformación de la experiencia. Ocurre automáticamente cada vez que el cerebro procesa información ya sea de percepciones, pensamientos, sentimientos, sensaciones o emociones (Spitzer, 2006), por lo que éste es un proceso dependiente de la experiencia, ya que ésta a su vez incide en cómo se van a percibir las nuevas experiencias. Así, el aprendizaje es un proceso continuo, individual, que se traduce en modos de pensar y actuar propios de cada individuo y además está fuertemente influido por el medio ambiente.

Sin embargo, una práctica de uso común observada en el ejercicio de la docencia, producto de una interpretación de la enseñanza centrada en el desarrollo de competencias, es la promoción y aplicación generalizada de metodologías activas, sin considerar los estilos de aprendizaje predominantes entre los estudiantes ni las diferencias debidas a los requerimientos específicos de las diferentes carreras y asignaturas. Como nos detallan García y Conde (2003: 80) «el objetivo es provocar un cambio de actitud de manera que sea el profesor quien adapte su estilo de enseñanza al estilo de aprendizaje preferente de sus alumnos».

Otra observación surgida de la práctica docente es que muchas decisiones en torno a las metodologías a emplear se toman actualmente sobre la base del ensayo y error, a partir de la aplicación de propuestas de tipo genérico, como, por ejemplo: aprender haciendo.

Sobre la base de estas consideraciones se escogió como objeto del presente estudio la caracterización de la variable Estilos de Aprendizaje que presentan estudiantes de diferentes carreras de la UM, pues es una variable que contribuye al conocimiento completo de ellos y debiera ser incluida en las metodologías innovadoras del siglo xxI, como una forma de vitalizar el proceso de enseñanza-aprendizaje (Gallego y García, 2012).

Ha de tenerse en cuenta que al hacer aparentes los estilos de aprendizaje se entrega una herramienta poderosa tanto a los docentes como a los estudiantes, para mejorar las habilidades de aprendizaje de éstos, logrando que aprendan a pensar mejor y más sabiamente (Järvelä, 2006), a la vez que se hacen más accesibles los conocimientos impartidos (Gallego y García, 2012) y se posibilita el desarrollar una comprensión de lo que se aprende tan profunda como sea posible.

\section{Metodología}

La presente investigación fue un estudio de carácter exploratorio en torno al objetivo general de si existen diferencias en los estilos de aprendizaje entre estudiantes de las jornadas diurna y vespertina en tres carreras de ingeniería de la UM. Este estudio cuantitativo presenta un diseño de tipo descriptivo, con alcances comparativos, no experimental y transversal y forma parte de una investigación 
MARCO ANTONIO DÍAZ DÍAZ E ISABEL ORTEGA SÁNCHEZ DE JORNADAS DIURNAS Y VESPERTINAS

más amplia, para la tesis doctoral Estilos de aprendizaje y métodos pedagógicos en la educación superior, centrada en la caracterización de los estilos de aprendizaje en una muestra de tamaño significativo y representativa, de 353 estudiantes, con un $5 \%$ de error (Sierra, 2007), a partir de un universo de 4.322 estudiantes de once carreras en cuatro facultades de la $\mathrm{UM}^{1}$.

El trabajo de investigación asociado a dicha tesis fue organizado en dos etapas, una primera cuantitativa orientada a caracterizar la incidencia de los diferentes estilos en las distintas carreras y una segunda cualitativa, en desarrollo, destinada a evaluar factores que incidan en el aprendizaje y estrategias de aprendizaje de los estudiantes. Este trabajo fue el producto de la etapa cuantitativa, y realizado en una subpoblación de 133 estudiantes seleccionada al azar de la muestra principal, de ambas jornadas de las carreras de Ingeniería Comercial (72), Civil Industrial (47) y Civil en Computación (14), manteniendo la proporcionalidad, de manera de conservar la representatividad de las distintas carreras (Sierra, 2007).

El instrumento mediante el cual se caracterizó la variable estilos de aprendizaje es el Cuestionario Honey Alonso de Estilos de Aprendizaje (CHAEA) (Alonso, Gallego y Honey, 2012), por ser un cuestionario adaptado para estudiantes universitarios de habla hispana y estar ampliamente validado, además de permitir el análisis estadístico y de correlación de variables. Se aplicó a estudiantes tanto de jornada diurna como vespertina con el fin de comparar los resultados y extraer conclusiones que arrojasen alguna luz sobre la pregunta planteada y que, también, permitan orientar y focalizar el proceso formativo de manera de contribuir a desarrollar metodologías más acordes a las características de aprendizaje predominantes entre los discentes.

Este cuestionario se configura en torno a la variable perfil de aprendizaje, la que cuenta con cuatro dimensiones, las que corresponden a estilos de aprendizaje o tendencias a desarrollar preferencias globales en la elección de estrategias para aprender. Las dimensiones son las siguientes: Activo, Reflexivo, Teórico y Pragmático. El instrumento consta de 80 declaraciones, organizadas de manera tal que a cada una de las cuatro dimensiones le corresponden veinte declaraciones, distribuidas de forma aleatoria, con las que se puede expresar acuerdo o desacuerdo mediante respuesta dicotómica. La validez y confiabilidad del instrumento fue reportada por los autores para cada uno de los estilos, con valores de $\alpha$-Cronbach: activo: 0.62; reflexivo: 0.72; teórico: 0.65, y pragmático: 0.58 (Alonso et al., 2012).

La recolección de datos se realizó mediante la aplicación de la encuesta en la población objetivo, estudiantes de las carreras previamente definidas, después de una charla de inducción, donde se explicaron brevemente los objetivos del estudio, se agradecía su colaboración y se daban garantías de conservación del anonimato y de la confidencialidad de la información entregada. El cuestionario se distribuyó a todos los asistentes a una clase, al inicio de la misma, que devolvían a medida

1. Fuente: U Mayor, SAP/BO, 30 de septiembre de 2013. 
que completaban, si es que decidían hacerlo pues hubo estudiantes que no lo hicieron. Este procedimiento fue acordado previamente con decanos, Direcciones de Escuela de las distintas carreras y con los profesores responsables de la clase donde se aplicaría la encuesta. Este procedimiento tuvo como ventaja el lograr la simultaneidad en el proceso de recolección de datos en cada nivel.

A la información recolectada en cada carrera se le asignó un código numérico, se transcribió y tabuló en una planilla de cálculo, en que cada línea refleja las respuestas de una encuesta y a cada respuesta dicotómica se le asignó un «1" o un «0» para, luego, agrupar la suma de las respuestas correspondientes a cada dimensión en una columna, configurándose así el perfil de aprendizaje para cada estudiante. Este proceso se repitió para cada encuesta, asignándosele a cada una un número identificador. Los datos así tabulados y ordenados fueron objeto de análisis estadístico mediante el programa STATA versión 13.

El primer paso del análisis estadístico fue comprobar si cada una de las dimensiones, en las distintas carreras, presentaba una distribución normal. Para ello se aplicó la prueba de Shapiro-Wilk, luego se realizaron pruebas de estadística descriptiva para realizar una estimación de las medias, desviación estándar y varianza y un análisis de frecuencias. Posteriormente, se realizó un estudio estadístico de relación de variables mediante la aplicación de la prueba de análisis de la varianza, ANOVA, para comparar las medias de las diferentes dimensiones en las distintas jornadas y la validez de la hipótesis nula que las medias no presentaban diferencias significativas en relación con la jornada.

\section{ANÁLISIS DE LOS RESULTADOS}

En primer lugar, se procedió a verificar la normalidad de la distribución de los puntajes de cada estilo de aprendizaje, no pudiéndose rechazar la hipótesis nula que todos seguían una distribución normal, por lo que se trabajó con promedios. Como se puede apreciar en la Tabla 1 no se aprecian grandes diferencias entre los estilos de aprendizaje de la jornada diurna y vespertina de una misma carrera.

A continuación, se efectuó la comparación grafica de los perfiles de aprendizaje para cada carrera en sus dos jornadas, y, como se puede apreciar en la Figura 1 , no se encontraron grandes diferencias entre los perfiles de aprendizaje en las jornadas diurna y vespertina de cada carrera, apreciación que se verificó con la aplicación de la prueba ANOVA. 
MARCO ANTONIO DÍAZ DÍAZ E ISABEL ORTEGA SÁNCHEZ

CARACTERIZACIÓN DE ESTILOS DE APRENDIZAJE EN ESTUDIANTES DE INGENIERÍA DE JORNADAS DIURNAS Y VESPERTINAS

TABLA 1

Puntaje promedio de cada Estilo de Aprendizaje en tres carreras de ingeniería, jornadas diurna y vespertina, U. Mayor, sede Santiago

\begin{tabular}{|c|c|c|c|c|c|}
\hline \multirow{2}{*}{ CARRERAS } & & Activo & REFLEXIVO & TEÓRICO & Pragmático \\
\hline & $\mathrm{N}^{(3)}$ & $\mathrm{M}(\mathrm{DE})$ & $\mathrm{M}(\mathrm{DE})$ & $\mathrm{M}(\mathrm{DE})$ & $\mathrm{M}(\mathrm{DE})$ \\
\hline & \multicolumn{5}{|c|}{ DIURNA } \\
\hline Ing. Comercial & 41 & $13(3)$ & $15(3)$ & $14(3)$ & $14(3)$ \\
\hline Ing. Civil Industrial & 39 & $11(3)$ & $15(3)$ & $15(2)$ & $13(3)$ \\
\hline \multirow[t]{2}{*}{ Ing. Civil Computación } & 11 & $12(3)$ & $16(2)$ & $13(2)$ & $14(3)$ \\
\hline & \multicolumn{5}{|c|}{ VESPERTINA } \\
\hline Ing. Comercial & 31 & $12(3)$ & $15(3)$ & $14(3)$ & $13(3)$ \\
\hline Ing. Civil Industrial & 8 & $13(4)$ & $16(3)$ & $16(2)$ & $15(2)$ \\
\hline \multirow[t]{2}{*}{ Ing. Civil Computación } & 3 & $11(3)$ & $16(2)$ & $14(2)$ & $12(1)$ \\
\hline & \multicolumn{5}{|c|}{ TOTAL } \\
\hline Ing. Comercial & 72 & $13(3)$ & $15(3)$ & $14(3)$ & $14(3)$ \\
\hline Ing. Civil Industrial & 47 & $12(3)$ & $15(3)$ & $15(2)$ & $14(3)$ \\
\hline Ing. Civil Computación & 14 & $12(3)$ & $16(2)$ & $13(2)$ & $14(3)$ \\
\hline
\end{tabular}

$\mathrm{M}=$ media, $\mathrm{DE}=$ desviación estándar.

${ }^{3}$ Fuente: U Mayor, SAP/BO, 30 de septiembre de 2013.

\section{FIGURA 1}

Comparación de los perfiles de aprendizaje de estudiantes de jornadas diurna y vespertina, en tres carreras de ingeniería de la U. Mayor, sede Santiago

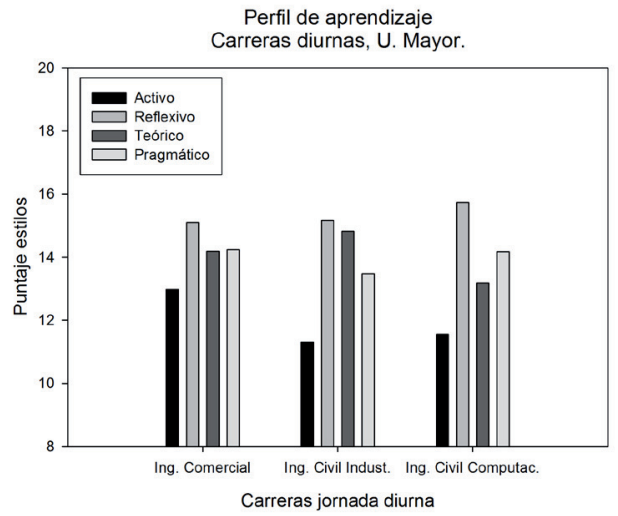

(C) Ediciones Universidad de Salamanca / CC BY-NC-ND

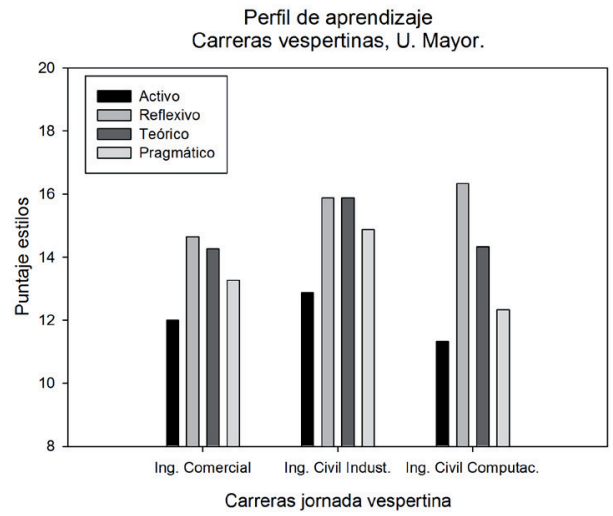

Enseñanza \& Teaching, 35, 2-2017, pp. 97-108 
Por otra parte, hay diferencias en la distribución de frecuencia del puntaje de los estilos de aprendizaje existiendo diferencias en la predominancia de estilos en las distintas carreras, como se puede apreciar en la Figura 2.

\section{FIGURA 2}

Muestra de la distribución de frecuencia de los puntajes que contribuyen al promedio en dos estilos de aprendizaje en tres carreras de ingeniería de la U. mayor, sede Santiago
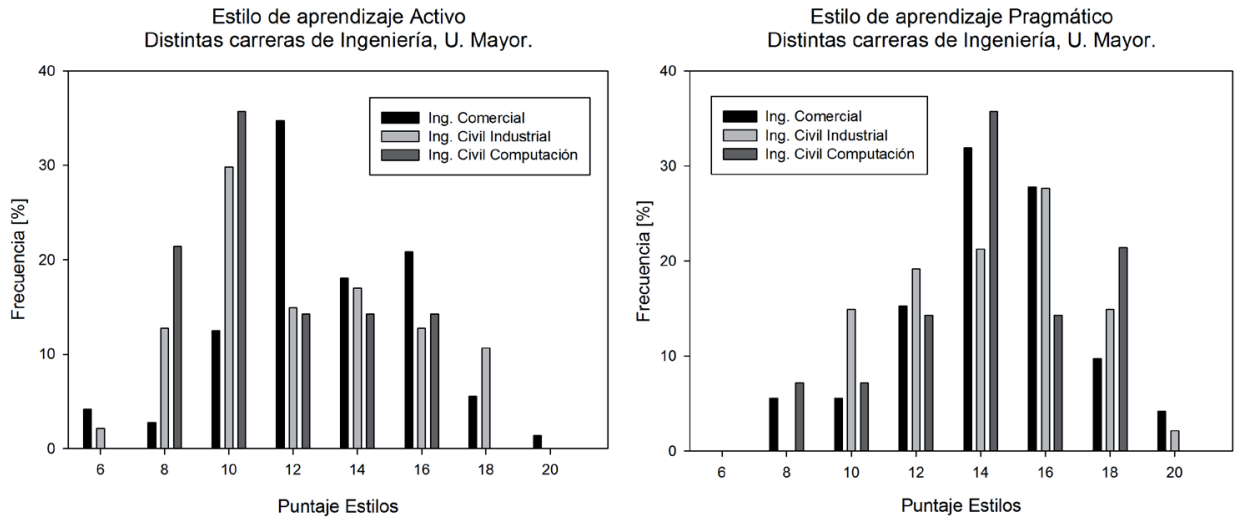

El paso siguiente fue comprobar la validez de la suposición que no existía una diferencia significativa entre las medias de los estilos de aprendizaje en las modalidades diurna y vespertina de una carrera mediante el análisis de las varianzas de los estilos de aprendizaje mediante ANOVA, considerando dos factores, jornada y carrera. Los valores obtenidos con esta prueba para el estadístico F y la probabilidad $p$ asociada fueron: 1.48 y 0.19 , respectivamente, por lo que se infirió que no había diferencia significativa entre los estilos de aprendizaje de los estudiantes que cursan estudios en jornadas diurna o vespertina. Lo cual se confirmó en el caso de los estudiantes de la carrera de Ingeniería Comercial al comparar las calificaciones de los grupos de jornada diurna y vespertina, puesto que el promedio general de ambos grupos difiere en sólo 0,1 punto.

Este resultado fue algo diferente de lo que se pudiera haber esperado, esto es, que los estudiantes de ambas jornadas hubieran desarrollado distintos estilos de aprendizaje para compensar en parte y por esta vía las diferencias que pudieran suscitarse por las diferentes exigencias al ingreso, tal como se comentó previamente. Sin embargo, esta suposición no fue avalada por los resultados expuestos previamente, por lo que resta encontrar otras explicaciones para sustentar la observación que el resultado académico, expresado en promedio general de notas, no fue diferente entre los estudiantes de las dos jornadas. Este planteamiento se sustenta sobre la condición de borde que las exigencias académicas eran iguales en ambas jornadas. 
MARCO ANTONIO DÍAZ DÍAZ E ISABEL ORTEGA SÁNCHEZ DE JORNADAS DIURNAS Y VESPERTINAS

\section{DISCUSIÓN Y CONCLUSIONES}

Los resultados aquí expuestos conducen al planteamiento de inquietudes tanto de forma como de fondo. Entre las primeras está la ratificación de los resultados de este estudio exploratorio, por ejemplo, ampliando la muestra, considerando como universo sólo las carreras con jornada vespertina, pero, dada la coherencia de los resultados obtenidos y la consistencia de las calificaciones, es probable que este resultado se mantenga.

En la misma línea, pero de una perspectiva cualitativa, estaría el indagar en las motivaciones y las formas de estudio que, desde la perspectiva de los actores, sustentan este exitoso esfuerzo de superación. Esto a partir del supuesto a priori, que, producto del ingreso especial que no exige la acreditación de conocimientos básicos mediante la rendición de la PSU ni las calificaciones de educación secundaria, estos estudiantes estarían en una situación de desventaja producto de deficiencias en conocimientos y competencias en materias básicas para la comprensión de las asignaturas propias de la carrera. También, habría que evaluar el rol que juegan la experiencia y la edad en esta nivelación de rendimiento, pues está comprobado que los recursos neurobiológicos involucrados en el aprendizaje varían con la edad (Spitzer, 2006).

Desde esta perspectiva y abordando un problema de fondo, otra línea de investigación guarda relación con el sistema de selección y los mecanismos para la construcción del puntaje de postulación a la educación superior, así como su congruencia con los requerimientos académicos conducentes a la adquisición de los conocimientos y competencias necesarios para la titulación y el desempeño profesional.

Tampoco se puede dejar de lado la consideración que los estudiantes de educación terciaria han dejado de ser niños, ingresan al sistema al término de la adolescencia y durante el curso de sus estudios se transforman en adultos, situación que es más marcada en la educación vespertina, donde el promedio de edad de ingreso es mayor que en la educación diurna, por lo que la conceptualización de las mallas curriculares y las metodologías debieran incorporar elementos de la educación para adultos, puesto que quienes participan en este proceso de enseñanza aprendizaje son adultos.

\section{REFERENCIAS BIBLIOGRÁFICAS}

Aguilera, E. (2012). Los estilos de enseñanza, una necesidad para la atención de los estilos de aprendizaje en la educación universitaria. Revista Estilos de Aprendizaje, 10, 79-87. Recuperado de http://www.uned.es/revistaestilosdeaprendizaje/numero_10/articulos/ Articulo07.pdf.

Alonso, C.; Gallego, D. y Honey, P. (2012). Los Estilos de aprendizaje. Procedimientos de diagnóstico y mejora (8. ${ }^{a}$ ed.). Bilbao, España: Mensajero.

Coffield, F.; Moseley, D.; Hall, E. y Eccleston, K. (2004). Learning styles and pedagogy in post-16 learning: A systematic and critical review. London, England: TheLearning and SkillsResearch Centre. 
MARCO ANTONIO DÍAZ DÍAZ E ISABEL ORTEGA SÁNCHEZ CARACTERIZACIÓN DE ESTILOS DE APRENDIZAJE EN ESTUDIANTES DE INGENIERÍA DE JORNADAS DIURNAS Y VESPERTINAS

De Miguel, M. (2005). Cambio de paradigma metodológico en la Educación Superior: Exigencias que conlleva. Cuadernos de Integración Europea, 2, 16-27. Recuperado de http://cde.uv.es/documents/2005-CIE-02.pdf.

Failla, I. (2010). Capital Humano: Nivel de educación de la fuerza de trabajo en Chile. Recuperado de http://biblioteca.duoc.cl/bdigital/observatorio/estudio/capital_humano.pdf.

Gallant, A. (2011). Resuscitating students' learning: Exploring the "Living Dead» phenomenon. The Educational Forum, 75 (4), 343-356. doi:10.1080/00131725.2011.602465.

Gallego, D. J. y García, M. C. (2012). Los estilos de aprendizaje en la formación inicial del docente. Rev. Estilos de Aprendizaje, 9, 4-20. Recuperado de http://www.uned.es/revistaestilosdeaprendizaje/numero_9/articulos/articulo1.pdf.

García, A. V. M. y Conde, M. J. R. (2003). Estilos de aprendizaje y educación superior: análisis discriminante en función del tipo de estudios. Enseñanza \& Teaching: Revista Interuniversitaria de Didáctica, 21, 77-97.

Järvelä, S. (2006). Personalized Learning? New Insights into Fostering Learning Capacity. En OECD Publishing. Personalizing Education. Schooling for Tomorrow (pp. 31-45). París, Francia: CERI/OECD.

Jiménez, M.; Lagos, F. y Durán, F. (2011). Propuestas para la educación superior. Foro Aequalis y las transformaciones necesarias. Santiago: Aequalis/INACAP.

Muñoz, P. y Redondo, A. (2013). Desigualdad y logro académico en Chile. Revista Cepal, 109, 107-123.

OECD (2009). Revisiones de Políticas Nacionales de Educación. La Educación Superior en Chile. París, Francia: OECD/IBRD/Banco Mundial.

OECD (2012). El Aseguramiento de la Calidad en la Educación Superior en Chile. París, Francia: OECD.

Sierra, R. (2007). Técnicas de investigación social. Teoría y ejercicios (14. ${ }^{a}$ ed.). Madrid, España: Thompson.

SIES/MINEDUC (2013). Informe Matrícula 2013. Recuperado de http://www.mifuturo.cl/index. php/informes-sies/matriculados.

Spitzer, M. (2006). Brain Research and Learning over the Life Cycle. En OECD Publishing. Schooling for Tomorrow. Personalizing Education (pp. 47-62). París, Francia: CERI/ OECD.

Taber, K. S. (2009). Constructivism and the crisis in U.S. science education: An essay review. Education Review, 12, 1-27. Recuperado de: http://www.edrev.info/essays/v12n12.pdf. 